\title{
Charged black holes in compactified spacetimes
}

\author{
Max Karlovin * and Rikard von Unge $\dagger$ \\ Department of Theoretical Physics and Astrophysics, Faculty of Science, \\ Masaryk University, Kotlar̆ská 2, 61137 Brno, Czech Republic
}

\begin{abstract}
We construct and investigate a compactified version of the four-dimensional ReissnerNordström-NUT solution, generalizing the compactified Schwarzschild black hole that has been previously studied by several workers. Our approach to compactification is based on dimensional reduction with respect to the stationary Killing vector, resulting in threedimensional gravity coupled to a nonlinear sigma model. Using that the original noncompactified solution corresponds to a target space geodesic, the problem can be linearized much in the same way as in the case of no electric nor NUT charge. An interesting feature of the solution family is that for nonzero electric charge but vanishing NUT charge, the solution has a curvature singularity on a torus that surrounds the event horizon, but this singularity is removed when the NUT charge is switched on. We also treat the Schwarzschild case in a more complete way than has been done previously. In particular, the asymptotic solution (the Levi-Civita solution with the height coordinate made periodic) has to our knowledge only been calculated up to a determination of the mass parameter. The periodic Levi-Civita solution contains three essential parameters, however, and the remaining two are explicitly calculated here.
\end{abstract}

PACS numbers: 04.40.Nr, 04.70.Bw, 11.25.Mj

\section{INTRODUCTION}

String theory predicts that our world has more dimensions than the 4 we see around us every day. The usual way out of this apparent discrepancy is to say that the additional dimensions are compactified and small. In the simplest case the extra dimensions are just circles. String theory is also a theory of gravity. It is therefore a natural and interesting question to ask what is the behavior of the classical solutions of General Relativity in spacetimes where one or more dimensions are compactified. This question is however surprisingly hard to answer. The periodic analogue of a Schwarzschild black hole in a space of topology $R 3 \times S 1$ has been st udied more or less independently by Majumdar [1], Papapetrou [2], Myers 3], Korotkin and Nicolai 4, 5] as well as by Frolov and Frolov 6]. In higher dimensions the full solution is known only implicitly 10, 12, 13, 20, 21].

The problem becomes even more interesting when one realizes, following Gregory and Laflamme 7, 8], that there is a competition between different gravitational configurations with the same mass and charges but with different symmetries and even of different horizon topology. Which configuration is stable depends on the particular value of the mass and the charges. For instance, a long and thin black string becomes unstable and will "decay" into a new configuration with higher entropy. This new configuration could for instance be a nontranslationally invariant black string or an infinite array of black holes. The phase diagram of black objects in higher dimensional circle space times has a very rich structure [1, 14, 16, 18]. Only parts of it is accessible to analytical methods and the most interesting pices has been calculated only numerically 17, 19, 22, 23, 24, 25] or using perturbation theory [15]. It would therefore clearly be of great interest to have a better analytical understanding of the various solutions that could appear.

In this paper we perform a more modest task. We generalize the solution given in $[3,[4,[6]$ to arbitrary electric and NUT charge. In doing so we find a new parameter of the solution which has not been recognized before. We analyze the physical meaning of this parameter. We also notice that adding NUT charge to the solution makes it more well behaved. Maybe this is a feature which would persist in higher dimensions? Our

\footnotetext{
*Electronic address: max@physics.muni.cz
}

$\dagger$ Electronic address: unge@physics.muni.cz 
method heavily relies on Weyl coordinates. However, as shown in 26], because of symmetry reasons, Weyl coordinates are expected to be useful at most in 4 and 5 dimensions. This is something which would have to be overcome if one would like to generalize our results to higher dimensions.

This paper is organized as follows. Section 2 is a general discussion of properties of the four dimensional Einstein-Maxwells equations important for our problem. In Section 3 we discuss the (nonperiodic) ReissnerNordström-NUT solution. In Section 4 we find its periodic generalization and discuss the metric in various limits. In Section 5 we give our conclusions.

\section{EINSTEIN-MAXWELLS EQUATIONS FOR STATIONARY SPACETIMES}

We write a general stationary metric with timelike Killing vector $\xi^{a}$ as

$$
g_{a b}=-f^{2} \mu_{a} \mu_{b}+f^{-2} h_{a b},
$$

where

$$
\begin{aligned}
& f=\sqrt{-\xi^{a} \xi_{a}} \\
& \mu_{a}=-f^{-2} \xi_{a}
\end{aligned}
$$

which implies that $h_{a b} \xi^{b}=0$ and that $f, \mu_{a}$ and $h_{a b}$ are stationary fields, i.e. Lie dragged by $\xi^{a}$. We now decompose the electromagnetic field strength $F_{a b}$ by defining the electric and magnetic fields $E_{a}$ and $B_{a}$ with respect to $\xi^{a}$ :

$$
\begin{aligned}
& E_{a}=F_{a b} \xi^{b} \\
& B_{a}=* F_{a b} \xi^{b}
\end{aligned}
$$

where $* F_{a b}$ is the dual field strength

$$
* F_{a b}=\frac{1}{2} \epsilon_{a b}{ }^{c d} F_{c d}
$$

It then follows that

$$
\begin{aligned}
F_{a b} & =2 E_{[a} \mu_{b]}-\epsilon_{a b}{ }^{c d} B_{c} \mu_{d} \\
* F_{a b} & =2 B_{[a} \mu_{b]}+\epsilon_{a b}{ }^{c d} E_{c} \mu_{d} .
\end{aligned}
$$

Assuming that $F_{a b}$ is stationary as well as source-free so that $* F_{a b}$ is closed, then both $E_{a}$ and $B_{a}$ are gradients of stationary scalars. Hence we introduce electric and magnetic potentials $v$ and $u$ according to

$$
E_{a}=-\nabla_{a} v, \quad B_{a}=-\nabla_{a} u .
$$

Moreover, with the electromagnetic field being the only matter source, a twist scalar $\chi$, likewise stationary, can be introduced by 34

$$
\nabla_{a} \chi=\epsilon_{a b c d} \xi^{b} \nabla^{d} \xi^{c}+2\left(u \nabla_{a} v-v \nabla_{a} u\right)
$$

The tensor $h_{a b}$ can be viewed as a metric on the three-dimensional manifold $\Sigma$ of Killing orbits and we shall use that the full 4D Einstein-Maxwell equations are now equivalent to the 3D equations obtained from the action

$$
S=\int d^{3} x \sqrt{h}\left[{ }^{(3)} R-2 h^{a b} \gamma_{A B} D_{a} X^{A} D_{b} X^{B}\right]
$$

where ${ }^{(3)} R, h^{a b}$ and $D_{a}$ are the Ricci scalar, inverse and Levi-Civita connection of $h_{a b}$. This action describes three-dimensional Euclidean gravity coupled to a certain sigma model. More precisely, the effective matter part of the action is that of a wave map from $\Sigma$ to a four-dimensional target space with metric

$$
d \sigma^{2}=\gamma_{A B} d X^{A} d X^{B}=\frac{\frac{1}{2} d \mathcal{E} d \overline{\mathcal{E}}+\psi d \bar{\psi} d \mathcal{E}+\bar{\psi} d \psi d \overline{\mathcal{E}}-(\mathcal{E}+\overline{\mathcal{E}}) d \psi d \bar{\psi}}{2\left[\frac{1}{2}(\mathcal{E}+\overline{\mathcal{E}})+\psi \bar{\psi}\right]^{2}}
$$


where $\mathcal{E}$ and $\psi$ are the complex Ernst potentials

$$
\mathcal{E}=f^{2}-v^{2}-u^{2}+i \chi, \quad \psi=v+i u .
$$

This is an Einstein metric, whose Ricci tensor obeys

$$
R_{A B}=-6 \gamma_{A B}
$$

If we use as target space coordinates $X^{A}$ the real variables $f, A, S$, $\chi$, where $A$ and $S$ are the amplitude and phase of $\psi$, i.e.

$$
\psi=A e^{i S}
$$

the target metric takes the neat form

$$
d \sigma^{2}=\frac{1}{f^{2}}\left(d f^{2}-d A^{2}-A^{2} d S^{2}\right)+\frac{\left(d \chi+2 A^{2} d S\right)^{2}}{4 f^{4}},
$$

which makes it evident that it has signature $(-,-,+,+)$. Now, varying the action (11) with respect to $h^{a b}$ gives

$$
{ }^{(3)} R_{a b}=2 \gamma_{A B} D_{a} X^{A} D_{b} X^{B},
$$

while variation with respect to the target space coordinates $X^{A}$ leads to the wave map equation

$$
h^{a b}\left(D_{a} D_{b} X^{C}+\Gamma_{A B}^{C} D_{a} X^{A} D_{b} X^{B}\right)=0,
$$

with $\Gamma_{A B}^{C}$ being the Christoffel symbols of $\gamma_{A B}$. For our purposes it is important to note that if the $X^{A}$ all depend solely on one free function $\omega$, then eqs. (17) and (18) become

$$
\begin{aligned}
& { }^{(3)} R_{a b}=2 \gamma_{A B} \frac{d X^{A}}{d \omega} \frac{d X^{B}}{d \omega} D_{a} \omega D_{b} \omega \\
& \frac{d X^{C}}{d \omega} h^{a b} D_{a} D_{b} \omega+\left(\frac{d^{2} X^{C}}{d \omega^{2}}+\Gamma_{A B}^{C} \frac{d X^{A}}{d \omega} \frac{d X^{B}}{d \omega}\right) h^{a b} D_{a} \omega D_{b} \omega=0 .
\end{aligned}
$$

Now, eq. (20) tells us that the curve $X^{A}(\omega)$ is a geodesic, since, as $h_{a b}$ is supposed to be a positive-definite metric, it cannot happen that $h^{a b} D_{a} \omega D_{b} \omega$ vanishes identically unless $\omega$ is constant. Using the freedom to reparametrize the geodesic, we can assume that it is affinely parametrized and arrive at the equations

$$
\begin{aligned}
& \epsilon:=\gamma_{A B} \frac{d X^{A}}{d \omega} \frac{d X^{B}}{d \omega}=\mathrm{constant} \\
& \frac{d^{2} X^{C}}{d \omega^{2}}+\Gamma_{A B}^{C} \frac{d X^{A}}{d \omega} \frac{d X^{B}}{d \omega}=0 \\
& { }^{(3)} R_{a b}=2 \epsilon D_{a} \omega D_{b} \omega \\
& h^{a b} D_{a} D_{b} \omega=0 .
\end{aligned}
$$

In this paper we focus exclusively on this class of solutions, which we shall refer to as geodesic solutions.

\section{A. Axisymmetry and Weyl coordinates}

Let us now assume that the stationary spacetime is also axisymmetric with an axisymmetry generator $\eta^{a}$ that commutes with the timelike Killing vector $\xi^{a}$. We can then introduce coordinates $(t, \rho, z, \phi)$ such that $\xi^{a}=(\partial / \partial t)^{a}, \eta^{a}=(\partial / \partial \phi)^{a}$ while the three-metric $h_{a b}$ and one-form $\mu_{a}$ take the forms

$$
\begin{aligned}
& d l^{2}=e^{2 k}\left(d \rho^{2}+d z^{2}\right)+W^{2} d \phi^{2} \\
& \boldsymbol{\mu}=d t+\Omega d \phi .
\end{aligned}
$$


The metric functions $f, k, W$ and $\Omega$ obviously depend on $\rho$ and $z$ only. If we assume that $\omega$ also depends solely on $\rho$ and $z$ (although it could have a linear dependence on $\phi$ with constant coefficient, a possibility which we do not consider here), the $\phi \phi$-component of eq. (23) becomes

$$
W_{, \rho \rho}+W_{, z z}=0
$$

making it possible to choose $\rho$ and $z$ such that $W=\rho$, a choice well-known as Weyl (canonical) coordinates. The great advantage of being able to use these coordinates is that the unknown metric function $k$ does not enter the Laplace equation (24) (nor the more general wave map equation (18)), which will thus be identical to the Laplace equation in flat space, expressed in cylindrical coordinates, for an unknown function that is independent of $\phi$;

$$
\omega_{, \rho \rho}+\rho^{-1} \omega_{, \rho}+\omega_{, z z}=0
$$

With the use of this equation, the remaining components of eq. (23) become

$$
\begin{aligned}
& k_{, \rho}=\epsilon \rho\left[\left(\omega_{, \rho}\right)^{2}-\left(\omega_{, z}\right)^{2}\right] \\
& k_{, z}=2 \epsilon \rho \omega_{, \rho} \omega_{, z},
\end{aligned}
$$

while eq. (10) gives

$$
\begin{aligned}
& \Omega_{, \rho}=4 p_{\chi} \rho \omega_{, z} \\
& \Omega_{, z}=-4 p_{\chi} \rho \omega_{, \rho},
\end{aligned}
$$

with $p_{\chi}$ being a constant equal to the conserved geodesic momentum associated with the cyclic coordinate $\chi$ of the target space metric. Explicitly,

$$
p_{\chi}=\frac{\frac{d \chi}{d \omega}+2 A^{2} \frac{d S}{d \omega}}{4 f^{4}} .
$$

A recipe for finding a solution of the type considered here is thus to choose an appropriate geodesic of the target space and an appropriate solution to the flat space Laplace equation (28). The geodesic then gives the four target space functions as functions of $\omega$, including the metric component $f$ and the potentials $v$ and $u$ which completely determine the electromagnetic field via eqs. (7) and (9). What then remains is to integrate the two pairs of equations (29) and (30) to determine the remaining spacetime metric components $k$ and $\Omega$. Note that eq. (28) is the integrability condition for both pairs of equations (when $\left.\epsilon \neq 0 \neq p_{\chi}\right)$.

\section{THE REISSNER-NORDSTRÖM-NUT SOLUTION}

Using Schwarzschild type coordinates, the Reissner-Nordström-NUT (RNN) solution with mass $M$, electric charge $Q$ and NUT (gravitational monopole) charge $l$ is

$$
\begin{array}{r}
d s^{2}=-f^{2}(d t+\Omega d \phi)^{2}+f^{-2} d r^{2}+\left(r^{2}+l^{2}\right)\left(d \theta^{2}+\sin ^{2} \theta d \phi^{2}\right), \\
\boldsymbol{F}=Q \frac{r^{2}-l^{2}}{\left(r^{2}+l^{2}\right)^{2}}(d t+\Omega d \phi) \wedge d r+\frac{2 Q l r}{r^{2}+l^{2}} \sin \theta d \theta \wedge d \phi .
\end{array}
$$

where

$$
\begin{aligned}
& f^{2}=\frac{r^{2}-2 M r+Q^{2}-l^{2}}{r^{2}+l^{2}}=\frac{\left(r-r_{+}\right)\left(r-r_{-}\right)}{r^{2}+l^{2}} \\
& \Omega=2 l \cos \theta+\Omega^{\prime} .
\end{aligned}
$$

Here we have defined

$$
r_{ \pm}=M \pm \Delta, \quad \Delta=\sqrt{M^{2}+l^{2}-Q^{2}},
$$


while $\Omega^{\prime}$ is a constant which should be set to $-2 l(2 l)$ to make the half-axis $\theta=0(\theta=\pi)$ explicitly regular, leaving the other half-axis - the Misner string - singular, as $d \phi$ is not a well-behaved one-form at $\theta=0, \pi$. However, it is well-known that both half-axes can be made regular, since changing $\Omega^{\prime}$ from $-2 l$ to $2 l$ can be mimicked by changing the time coordinate from $t$ to $t^{\prime}=t-4 l \phi$. The price to pay is closed timelike curves since it requires that $t$ and $t^{\prime}$ should both be periodic with period $8 \pi l$.

The three-metric $h_{a b}$ for this solution is seen to be

$$
d l^{2}=d r^{2}+\left(r^{2}-2 M r+Q^{2}-l^{2}\right) d \Omega^{2}=d r^{2}+\left(r-r_{+}\right)\left(r-r_{-}\right) d \Omega^{2}
$$

and the three remaining target space scalars are given by

$$
\begin{aligned}
\chi & =\frac{2 l(r-M)}{r^{2}+l^{2}} \\
A & =\frac{Q}{\sqrt{r^{2}+l^{2}}} \\
S & =-\arctan \left(\frac{l}{r}\right),
\end{aligned}
$$

Since all the $X^{A}$ depend on $r$ only, this is a geodesic solution. In the non-extremal case $\Delta>0$, the geodesic is spacelike $(\epsilon>0)$ and explicitly given by

$$
\begin{aligned}
& f=\frac{\Delta}{\sqrt{(M \sinh \bar{\omega}-\Delta \cosh \bar{\omega})^{2}+l^{2} \sinh ^{2} \bar{\omega}}}=\frac{r_{+}-r_{-}}{\sqrt{\left(r_{-} e^{\bar{\omega}}-r_{+} e^{-\bar{\omega}}\right)^{2}+l^{2}\left(e^{\bar{\omega}}-e^{-\bar{\omega}}\right)^{2}}} \\
& \chi=-\frac{l \Delta \sinh 2 \bar{\omega}}{(M \sinh \bar{\omega}-\Delta \cosh \bar{\omega})^{2}+l^{2} \sinh ^{2} \bar{\omega}}=-\frac{2 l \Delta\left(e^{2 \bar{\omega}}-e^{-2 \bar{\omega}}\right)}{\left(r_{-} e^{\bar{\omega}}-r_{+} e^{-\bar{\omega}}\right)^{2}+l^{2}\left(e^{\bar{\omega}}-e^{-\bar{\omega}}\right)^{2}} \\
& A=-\frac{Q \sinh \bar{\omega}}{\sqrt{(M \sinh \bar{\omega}-\Delta \cosh \bar{\omega})^{2}+l^{2} \sinh ^{2} \bar{\omega}}}=-\frac{Q\left(e^{\bar{\omega}}-e^{-\bar{\omega}}\right)}{\sqrt{\left(r_{-} e^{\bar{\omega}}-r_{+} e^{-\bar{\omega}}\right)^{2}+l^{2}\left(e^{\bar{\omega}}-e^{-\bar{\omega}}\right)^{2}}} \\
& S=-\arctan \left(\frac{l \sinh \bar{\omega}}{M \sinh \bar{\omega}-\Delta \cosh \bar{\omega}}\right)=-\arctan \left[\frac{l\left(e^{\bar{\omega}}-e^{-\bar{\omega}}\right)}{r_{-} e^{\bar{\omega}}-r_{+} e^{-\bar{\omega}}}\right],
\end{aligned}
$$

where $\bar{\omega}$ is the arclength parameter for the geodesic, and here the function that satisfies the Laplace equation (24), namely

$$
\bar{\omega}=-\operatorname{arccoth}\left(\frac{r-M}{\Delta}\right)=\frac{1}{2} \ln \left(\frac{r-r_{+}}{r-r_{-}}\right) .
$$

However, we shall instead think of the geodesic as parametrized by the rescaled affine parameter

$$
\omega=\frac{M}{\Delta} \bar{\omega}
$$

which implies that the norm of the geodesic tangent vector has norm $\epsilon=\Delta / M$. The reason for this is that we will then straightforwardly be able to treat the extremal case - which corresponds to a null geodesic $(\epsilon=0)$ - as the $\Delta \rightarrow 0$ limit of the non-extremal case. Indeed, taking this limit for the above formulae, we find that the spacelike geodesic goes over into a lightlike one;

$$
\begin{aligned}
& f=\frac{M}{\sqrt{M^{2}(1-\omega)^{2}+l^{2} \omega^{2}}} \\
& \chi=-\frac{2 l M \omega}{M^{2}(1-\omega)^{2}+l^{2} \omega^{2}} \\
& A=-\frac{Q \omega}{\sqrt{M^{2}(1-\omega)^{2}+l^{2} \omega^{2}}} \\
& S=\arctan \left[\frac{l \omega}{M(1-\omega)}\right],
\end{aligned}
$$


while $\omega$ goes over into

$$
\omega=-\frac{M}{r-M}
$$

This limiting procedure would not have worked, had we used $\bar{\omega}$ as the affine parameter. Moreover, $\omega$ has the large $r$ asymptotic behaviour

$$
\omega=-\frac{M}{r}+O\left(r^{-2}\right)
$$

which means that it is $\omega$, rather than $\bar{\omega}$, that corresponds to a Newtonian gravitational potential. However, although we will think of the geodesic as parametrized by $\omega$, we will often work with $\bar{\omega}$ in subsequent calculations, since the factor $M / \Delta$ would otherwise often appear merely as an annoying appendage.

For future reference, we here finally calculate the conserved geodesic momentum $p_{\chi}$ according to the formula (31), to find the simple relation

$$
p_{\chi}=-\frac{l}{2 M}
$$

\section{A. Charges}

A special and somewhat surprising feature of the RNN solution is that although no sources are included for the electromagnetic field, the result of calculating the electric charge $Q_{\mathrm{el}}$ by integrating $* F_{a b}$ over a twosphere of constant $t$ and $r$ and dividing by $4 \pi$ is not what one would naively expect from Stoke's theorem, i.e. the result is not independent of the choice of two-sphere but depends on the radius;

$$
Q_{\mathrm{el}}=Q \frac{r^{2}-l^{2}}{r^{2}+l^{2}}
$$

We also calculate the magnetic charge $Q_{\text {mag }}$ by integrating $F_{a b}$ over the same sphere to find

$$
Q_{\mathrm{mag}}=\frac{2 Q l r}{r^{2}+l^{2}}
$$

Obviously we have $Q_{\mathrm{el}}=Q, Q_{\mathrm{mag}}=0$ asymptotically as $r \rightarrow \infty$, so at infinity the solution is purely electric with charge $Q$. It is worth noting that the relation

$$
Q_{\mathrm{el}}^{2}+Q_{\mathrm{mag}}^{2}=Q^{2}
$$

holds as an identity, for all $r$. The reason why it is possible that electric and magnetic charges depend on $r$ is that the gravitomagnetic vector potential $\Omega d \phi$ enters not only the metric but also the electromagnetic field strength (33). Thus the latter is singular on the Misner string, i.e. on $\theta=0$ or $\theta=\pi$ (or both) depending on how $\Omega^{\prime}$ is chosen. As the Misner string goes through every two-sphere that we integrate over, we cannot expect Stoke's theorem to imply that the charges should be independent of the choice of sphere. Interestingly, if we refrain from removing the singularity by introducing periodic time, the Misner string must be thought of as a concrete physical object as it carries electric and magnetic charge density.

\section{B. Transformation to Weyl coordinates}

For the RNN solution, the transformation from Schwarzschild type coordinates to Weyl coordinates reads

$$
\begin{aligned}
& \rho=\sqrt{(r-M)^{2}-\Delta^{2}} \sin \theta \\
& z=(r-M) \cos \theta
\end{aligned}
$$


with inverse transformation

$$
\begin{aligned}
& r=\lambda+M \\
& \cos \theta=\frac{z}{\lambda},
\end{aligned}
$$

where

$$
\lambda=\frac{1}{2}\left(\lambda_{+}+\lambda_{-}\right), \quad \lambda_{ \pm}=\sqrt{\rho^{2}+(z \pm \Delta)^{2}} .
$$

The functions $\omega, k$ and $\Omega$ are now given by

$$
\begin{aligned}
\omega & =\frac{M}{2 \Delta} \ln \left(\frac{\lambda-\Delta}{\lambda+\Delta}\right) \\
k & =\frac{1}{2} \ln \left(\frac{\lambda^{2}-\Delta^{2}}{\lambda^{2}-\eta^{2}}\right), \quad \eta=\frac{1}{2}\left(\lambda_{+}-\lambda_{-}\right) \\
\Omega & =\frac{2 l z}{\lambda}+\Omega^{\prime} .
\end{aligned}
$$

In the non-extremal case $\Delta>0$, the function $\omega$ is the potential of an infinitely thin rod located at $\rho=0$, $|z| \leq \Delta$ and having a line density $M /(2 \Delta)$ per unit length, which means that the line density can be interpreted as the mass line density. Note that in the extremal case $\Delta=0$, the function $k$ vanishes identically

leaving the three-metric $h_{a b}$ flat. Moreover $\lambda$ reduces to $\sqrt{\rho^{2}+z^{2}}$ and $\omega$ to the standard monopole solution $-M / \lambda$.

\section{PERIODIC ANALOGUE OF THE REISSNER-NORDSTRÖM-NUT SOLUTION}

We will now construct compactified versions of the RNN solution, which generalize the compactified Schwarzschild solution discussed in [3, 4, 5, 6]. The approach we take can be summerized as follows:

- For every member of the RNN family of solutions, compactify the function $\omega$ by using canonical Weyl coordinates and taking the $z$-periodic analogue of the original solution to the Laplace equation (28). This is straightforward due to the linearity of the latter.

- Insert $\omega$ into the same geodesic that defines the original RNN solution.

- Integrate eqs. (29) and (30) to find the remaining metric functions $k$ and $\Omega$.

Before we start, some general remarks about the first of these steps are in order. As proved by Korotkin and Nicolai [4, 5], if ${ }^{0} \omega(\rho, z)$ is a solution to the Laplace equation with the asymptotic behaviour

$$
{ }^{0} \omega(\rho, z)=-\frac{M}{\tilde{r}}+O\left(\tilde{r}^{-2}\right) \quad \text { as } \tilde{r} \rightarrow \infty
$$

where $\tilde{r}=\sqrt{\rho^{2}+z^{2}}$ and $M$ is some constant (in our case the mass), then the series

$$
\omega(\rho, z)=\sum_{n=-\infty}^{\infty}\left[{ }^{0} \omega(\rho, z+n L)+a_{n}\right], \quad a_{n}= \begin{cases}\frac{M}{L|n|} & \text { if } n \neq 0 \\ 0 & \text { if } n=0\end{cases}
$$

is convergent for any $(\rho, z)$ such that $(\rho, z+n L)$ does not coincide with a singular point of ${ }^{0} \omega(\rho, z)$ for any integer $n$. The resulting function $\omega$ then obviously defines a $z$-periodic solution to the Laplace equation (28) with period $L$. The constants $a_{n}$ are essential as they make the series converge, but one cannot say that 
they are uniquely determined as one could add to $a_{n}$ any other $n$-dependent constant $b_{n}$ that falls off faster than $|n|^{-1}$ as $|n| \rightarrow \infty$ so that

$$
B:=\sum_{n=-\infty}^{\infty} b_{n}
$$

is a finite constant. In other words, the $z$-periodic analogue of the function ${ }^{0} \omega(\rho, z)$ is a priori only defined up to an additive constant $B$. Korotkin and Nicolai fix this constant by taking eq. (66) as it stands $(B=0)$ to define $\omega(\rho, z)$, but this choice has not been made by the other workers that have studied the compactified Schwarzschild black hole; while Myers [3] makes an explicitly different choice of $a_{n}$ leading to a certain nonzero $B$, Frolov and Frolov $\underline{6}$ instead use a Green's function method to compactify which in effect corresponds to a third choice of $B$. Now, since the function $\omega(\rho, z)$ is periodic in $z$, it does not make sense to study its behaviour for large $\tilde{r}$, but for large $\rho$ it has the asymptotic behaviour

$$
\omega=\frac{2 M}{L} \ln \rho+O(1)
$$

If $\omega$ would tend to a constant in this limit, it would be natural to make that constant vanish by a suitable choice of $B$. However, since $\omega$ instead diverges logarithmically (unless $M=0$ ), we see no physical motivation for fixing $B$ at any particular value and hence we will keep it as a free parameter.

We will now implement the compactification scheme as outlined above. We thus take ${ }^{0} \omega$ to be the function given by eq. (62). According to the above, its periodic analogue is

$$
\omega=\sum_{n=-\infty}^{\infty}\left[\frac{M}{2 \Delta} \ln \left(\frac{\lambda_{n}-\Delta}{\lambda_{n}+\Delta}\right)+a_{n}\right]+B
$$

where

$$
\begin{aligned}
& \lambda_{n}=\frac{1}{2}\left[\sqrt{\rho^{2}+\left(z_{n}+\Delta\right)^{2}}+\sqrt{\rho^{2}+\left(z_{n}-\Delta\right)^{2}}\right], \quad z_{n}=z-n L \\
& a_{n}= \begin{cases}\frac{M}{L|n|}, & \text { if } n \neq 0 \\
0 & \text { if } n=0 .\end{cases}
\end{aligned}
$$

We take $z$ to have the range $z \in[-L / 2, L / 2]$ with the end points of the interval identified. The horizon, where $\omega$ diverges to minus infinity, will thus be located at $\rho=0,|z| \leq \Delta$, just as in the non-compactified case. Since we shall not consider the case when the horizon overlaps itself, we require that $\Delta \leq L / 2$. It will prove useful to introduce the dimensionless variable

$$
\beta=\frac{2 \Delta}{L},
$$

having the range $\beta \in[0,1]$. For convenience, we will, for the time being, work with $\bar{\omega}$ and use the dimensionless coordinates

$$
x=\frac{\rho}{\Delta}, \quad y=\frac{z}{\Delta} .
$$

Clearly $\bar{\omega}$ is then given by

$$
\bar{\omega}=\sum_{n=-\infty}^{\infty}\left[\frac{1}{2} \ln \left(\frac{\lambda_{n}^{\prime}-1}{\lambda_{n}^{\prime}+1}\right)+\bar{a}_{n}\right]+\bar{B}
$$


where

$$
\begin{aligned}
& \lambda_{n}^{\prime}=\frac{1}{2}\left[\sqrt{x^{2}+\left(y_{n}+1\right)^{2}}+\sqrt{x^{2}+\left(y_{n}-1\right)^{2}}\right], \quad y_{n}=y-2 n \beta^{-1} \\
& \bar{a}_{n}=\frac{\Delta}{M} a_{n}= \begin{cases}\frac{\beta}{2|n|}, & \text { if } n \neq 0 \\
0 & \text { if } n=0,\end{cases} \\
& \bar{B}=\frac{\Delta}{M} B .
\end{aligned}
$$

We shall now explicitly evaluate $\bar{\omega}$ close to the symmetry axis $x=0$. As the behaviour on the horizon section $|y| \leq 1$ of the axis is different from the off-horizon section $1<|y| \leq \beta^{-1}$, these two sections will have to be treated differently, but in both cases we shall express the result in terms of the function

$$
\varphi_{\beta}(\xi)=\frac{\beta}{2 \pi} \sin \left(\frac{\pi \beta \xi}{2}\right) \Gamma\left(\frac{\beta \xi}{2}\right)^{2}
$$

having the properties

$$
\begin{aligned}
& \lim _{\beta \rightarrow 0} \varphi_{\beta}(\xi)=\frac{1}{\xi} \\
& \varphi_{\beta}(\xi) \varphi_{\beta}(-\xi)=-\frac{1}{\xi^{2}} .
\end{aligned}
$$

Moreover, for reasons that will become clear, we shall replace the free constant $\bar{B}$ by a free constant $\bar{u}$ according to

$$
\bar{B}=\bar{u}-\beta \gamma-\frac{1}{2} \ln \left[2 \varphi_{\beta}(2)\right],
$$

where $\gamma$ is the Euler constant. Now, for small $x$ and $|y| \leq 1$, we find that $\bar{\omega}$ is given by

$$
e^{2 \bar{\omega}}=\frac{e^{2 \bar{u}}}{4 H(y)} x^{2}+O\left(x^{4}\right),
$$

where $H(y)$ is the function

$$
H(y)=\frac{2 \varphi_{\beta}(2)}{\varphi_{\beta}(1+y) \varphi_{\beta}(1-y)} .
$$

For $1<|y| \leq \beta^{-1}$, on the other hand, one finds that

$$
e^{2 \bar{\omega}}=\frac{e^{2 \bar{u}}}{2 \varphi_{\beta}(2)} \frac{\varphi_{\beta}(|y|+1)}{\varphi_{\beta}(|y|-1)}+O\left(x^{2}\right) .
$$

This is all that is needed to generate the whole solution. Indeed, for both axis sectors, $\bar{\omega}$ is a solution to the Laplace equation which can be expanded in terms of a constant $C$ and a function $A(y)$ as

$$
\bar{\omega}=C \ln x+\sum_{k=0}^{\infty} \frac{(-1)^{k}}{\left(2^{k} k !\right)^{2}} \frac{d^{2 k} A(y)}{d y^{2 k}} x^{2 k} .
$$

Inserting this expansion into the right hand sides of eqs. (29) and (30) (using eq. (53) for the latter), we find that $k$ and $\Omega$ are determined up to additive constants $k^{\prime}$ and $\Omega^{\prime}$;

$$
\begin{aligned}
& k=k^{\prime}+C^{2} \ln x+2 C A(y)-\frac{1}{2}\left[C A^{\prime \prime}(y)+A^{\prime}(y)^{2}\right] x^{2}+O\left(x^{4}\right) \\
& \Omega=\Omega^{\prime}+2 l\left[C y+\sum_{k=1}^{\infty} \frac{2 k(-1)^{k}}{\left(2^{k} k !\right)^{2}} \frac{d^{2 k-1} A(y)}{d y^{2 k-1}} x^{2 k}\right]
\end{aligned}
$$


where we refrain from giving the whole series for $k$ since it depends nonlinearly on $\bar{\omega}$, although it would not be difficult to write down at least a few more terms. The value of $k^{\prime}$ has to be determined from the requirement that the axis be regular. Starting with the off-horizon section, regularity means absence of a conical singularity which is in turn means that $k$ should vanish as $x \rightarrow 0$, at least if we assume that $\Omega$ vanishes in that limit, which can always be achieved as we shall see below. Thus, since the constant $C$ is zero in this case, we directly obtain $k^{\prime}=0$. For the horizon section we instead have $C=1$. In this case we may determine $k^{\prime}$ by inspecting the horizon metric, which reads

$$
d s_{\mathrm{H}}^{2}=R^{2}\left[\frac{e^{2\left(k^{\prime}+\bar{u}\right)}}{16} \frac{d y^{2}}{H(y)}+H(y) d \phi^{2}\right],
$$

while $R$ is the constant

$$
R=\sqrt{r_{+}^{2}+l^{2}} e^{-\bar{u}}
$$

Now, since

$$
H^{\prime}( \pm 1)=\mp 2
$$

it follows that for the horizon's polar points $y= \pm 1$ to be regular, we must set

$$
k^{\prime}=-\bar{u}+\ln 4 \text {. }
$$

When we are on the horizon $|y| \leq 1$, the constant $\Omega^{\prime}$ in eq. (87) precisely corresponds to the constant $\Omega^{\prime}$ in eqs. (35) and (64) and should be set to $-2 l(2 l)$ to make $\Omega$ vanish at the horizon pole $y=1(y=-1)$. Continuity at $y= \pm 1$ then requires us to set $\Omega^{\prime}=0$ for $y>1(y<1)$ and $\Omega^{\prime}=-4 l\left(\Omega^{\prime}=4 l\right)$ for $y<1$ $(y>1)$. Again, these two choices do not correspond to different physics, but are related by a change of time $t \rightarrow t^{\prime}=t-4 l \phi$ which results in time periodicity with the period $8 \pi l$. However, note that unlike the non-periodic case, both choices imply that $\Omega$ has a jump discontinuity where $y=\beta^{-1}$ is periodically identified with $y=-\beta^{-1}$. This discontinuity is of course not of a physical nature either, since we can shift it to any other position on the $y$-axis. For instance, we may put the discontinuity somewhere on the horizon and obtain a completely regular off-horizon section on which $\Omega$ is everywhere zero.

\section{A. Fourier expansions}

In principle, the full RNN solution is given as the $x=0$ expansions (85) - (87), but since these series converge very slowly for large $x$ and are quite useless when it comes to determining the asymptotic behaviour as $x \rightarrow \infty$, we shall now follow Frolov and Frolov and represent the solution in terms of fourier series, thus making explicit use of the periodicity of the coordiante $y$. Still using the rescaled coordinates $(x, y)$, we assume fourier expansions for the functions $\bar{\omega}$ and $k$ of the forms

$$
\begin{aligned}
& \bar{\omega}=\sum_{q=0}^{\infty} \bar{\omega}_{q}(x) \cos (\pi q \beta y) \\
& k=\sum_{q=0}^{\infty} k_{q}(x) \cos (\pi q \beta y)
\end{aligned}
$$

Since $\bar{\omega}$ is a solution to the Laplace equation (28), it follows that

$$
\bar{\omega}_{q}^{\prime \prime}(x)+x^{-1} \bar{\omega}_{q}^{\prime}(x)-(\pi q \beta)^{2} \bar{\omega}_{q}(x)=0 .
$$

Disqualifying solutions that diverges exponentially as $x \rightarrow \infty$, we obtain that $\bar{\omega}_{0}(x)$ is a linear function of $\ln x$ while, for $q>0$,

$$
\bar{\omega}_{q}(x) \propto K_{0}(\pi q \beta x)
$$


where $K_{0}$ is the modified Bessel function of the second kind. For small $x$, we have

$$
-K_{0}(\pi q \beta x)=\gamma+\ln \left(\frac{\pi q \beta x}{2}\right)+O\left(x^{2}\right) .
$$

We can now use that we already know $\bar{\omega}$ for small $x$ to find that

$$
\begin{aligned}
& \bar{\omega}_{0}(x)=\bar{u}-\frac{1}{2} \ln \left[2 \varphi_{\beta}(2)\right]+\beta \ln \left(\frac{\beta x}{4}\right) \\
& \bar{\omega}_{q}(x)=-\frac{2}{\pi q} \sin (\pi q \beta) K_{0}(\pi q \beta x) \text { for } q>0 .
\end{aligned}
$$

We use this fourier expansion for $\bar{\omega}$ to plot the metric function $f$ for two choices of the parameters $M, Q$ and $l$ (figure 1).

Due to the nonlinearity of eqs. (29) which governs $k$, we shall not attempt to give its full fourier series. However, since all $k_{q}(x)$ with $q>0$ decay exponentially as $x \rightarrow \infty$, it will suffice to determine $k_{0}(x)$ to obtain the asymptotic behaviour in that limit. Now, the zeroth order fourier term of the first of eqs. (29) reads

$$
k_{0}^{\prime}(x)=x\left[\left(\bar{\omega}_{, x}\right)^{2}-\left(\bar{\omega}_{, z}\right)^{2}\right]_{0}=x\left\{\bar{\omega}_{0}^{\prime}(x)^{2}+\frac{1}{2} \sum_{q=1}^{\infty}\left[\bar{\omega}_{q}^{\prime}(x)^{2}-(\pi q \beta)^{2} \bar{\omega}_{q}(x)^{2}\right]\right\}
$$

Inserting the determined expressions for the $\bar{\omega}_{q}(x)$ gives

$$
k_{0}^{\prime}(x)=\beta^{2} x\left\{\frac{1}{x^{2}}+2 \sum_{q=1}^{\infty} \sin ^{2}(\pi q \beta)\left[K_{1}(\pi q \beta x)^{2}-K_{0}(\pi q \beta x)^{2}\right]\right\}
$$

which can be integrated to yield

$$
k_{0}(x)=k_{*}+\beta^{2}\left\{\ln x+x^{2} \sum_{q=1}^{\infty} \sin ^{2}(\pi q \beta)\left[2 K_{1}(\pi q \beta x)^{2}-K_{0}(\pi q \beta x) K_{2}(\pi q \beta x)-K_{0}(\pi q \beta x)^{2}\right]\right\},
$$

where $k_{*}$ is some constant yet to be determined. Using now that

$$
x^{2}\left[2 K_{1}(\pi k \beta x)^{2}-K_{0}(\pi k \beta x) K_{2}(\pi k \beta x)-K_{0}(\pi k \beta x)^{2}\right]=\frac{2}{(\pi k \beta)^{2}}\left[1+\gamma+\ln \left(\frac{\pi k \beta x}{2}\right)\right]+O\left(x^{2}\right),
$$

we can compare $k_{0}(x)$ as given by eq. (101) to the zeroth order fourier term of the exact expression for $k$ we have near $x=0$ to find, after some manipulations, that $k_{*}$ is the $\beta$-dependent constant

$$
k_{*}=\beta^{2}\left[1+\ln \left(\frac{\beta}{4}\right)\right]-\beta \ln \left[\varphi_{\beta}(2)\right]+\int_{0}^{\beta} \ln \left[\varphi_{\beta^{\prime}}(2)\right] d \beta^{\prime} .
$$

Finally, from eqs. (30) and (53) we obtain that $\Omega$ is given by the following "quasi-fourier" series:

$$
\Omega=\Omega^{\prime}+2 l \beta y+4 l \sum_{k=1}^{\infty} \frac{1}{\pi k} \sin (\pi k \beta) x K_{1}(\pi k \beta x) \sin (\pi k \beta y)
$$

where $\Omega^{\prime}$ is the same constant that appears in eq. (87) for the case $|y| \leq 1$.

\section{B. Properties of the event horizon}

As noted above, the (outer) event horizon has the geometry

$$
d s_{\mathrm{H}}^{2}=\mathcal{R}^{2}\left[\frac{d y^{2}}{H(y)}+H(y) d \phi^{2}\right], \quad \mathcal{R}=e^{-\bar{u}} \sqrt{r_{+}^{2}+l^{2}} .
$$



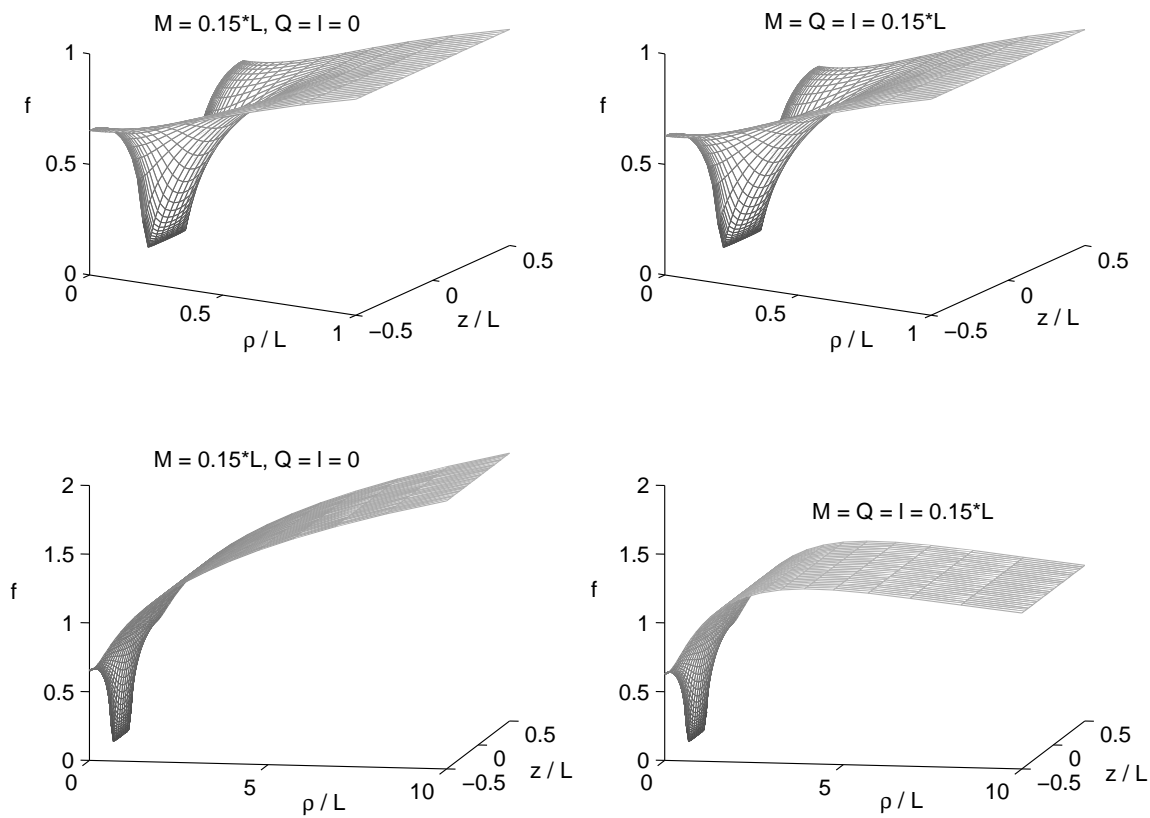

FIG. 1: The metric function $f=\sqrt{-g_{t t}}$, plotted for $M=0.15 L, Q=l=0$ as well as for $M=Q=l=0.15 L$, with $\bar{u}=0$ in both cases. As the two top plots show, the behaviour of $f$ for the two cases is very similar close to the axis $\rho=0$. On the other hand $f$ behaves qualitatively different when a larger range of $\rho$ is included, as indicated by the plots below; in the first case $f$ increases monotonically with $\rho$ towards infinity, while in the second case $f$ reaches a maximum and subsequently decreases towards zero. The latter behaviour is generic as long as $l \neq 0$, regardless of $Q$, but with $l=0 \neq Q$, the maximum is replaced by a divergence, as discussed in subsection IVD

It directly follows that the horizon area and Gaussian curvature (half the Ricci scalar) are given by the simple formulae

$$
\begin{aligned}
& \mathcal{A}=4 \pi \mathcal{R}^{2} \\
& K=-\frac{1}{2} H^{\prime \prime}(y) \mathcal{R}^{-2} .
\end{aligned}
$$

It is worth noting that $H(y)$ behaves for small $\beta$ as

$$
H(y)=1-y^{2}-\frac{1}{2} \zeta(3)\left(1-y^{2}\right)^{2} \beta^{3}+O\left(\beta^{5}\right),
$$

while for $\beta$ close to unity it behaves as

$$
H(y)=4(1-\beta)+4\left[\Psi\left(\frac{1+y}{2}\right)+\Psi\left(\frac{1-y}{2}\right)+2 \gamma+1\right](1-\beta)^{2}+O\left((1-\beta)^{3}\right),
$$

where $\Psi$ is the digamma function (the logarithmic derivative of the gamma function). Clearly, for $\beta=0$, the metric is an exact two-sphere of radius $\mathcal{R}$. Note that $\beta \rightarrow 0$ corresponds to two independent and physically different limits, namely the extremal limit $\Delta \rightarrow 0$ as well as the limit of infinite coordinate diameter $L \rightarrow \infty$. This is natural since both limits, makes the proper distance between the poles of the black hole infinite, keeping the black hole from being distorted "by itself", i.e. by the gravitational field from its periodic 
copies. As $\beta$ is increased, the sphere becomes deformed in a prolate manner. For small $\beta$ the deformation is very small, however, as the first correction term in eq. (108) enters at order $\beta^{3}$. As $\beta$ is further increased the deformed sphere gets stretched out to a long cigar, and for $\beta$ close to unity the geometry is almost everywhere a flat cylinder of length $\mathcal{R} / \sqrt{1-\beta}$ and circumference $4 \pi \mathcal{R} \sqrt{1-\beta}$, except at the poles $y= \pm 1$ where the curvature is very high (note that the mean Gaussian curvature is always $\mathcal{R}^{-2}$ ). Up to a constant conformal factor the geometry only depends on the parameter $\beta$ and hence we can refer the reader to Frolov and Frolov 6 for a further discussion of its properties, as well as embedding diagrams. Now, the horizon area $\mathcal{A}$ is clearly modified by a factor $e^{-2 \bar{u}}$ compared to the non-compactified case. This is in agreement with the general treatment of distorted black holes with (electric) charge as presented by Fairhurst and Krishnan 27], based on Geroch and Hartle's treatment of the case without charge 28]. Although it may not have been obvious from the way we introduced it, the parameter $\bar{u}$ (denoted the same way in [27] but with an unbarred $u$ in [28] and [6]) can be defined as

$$
\bar{u}=\left.\delta \bar{\omega}\right|_{\rho=0, z= \pm \Delta}, \quad \delta \bar{\omega}=\bar{\omega}-{ }^{0} \bar{\omega}
$$

i.e. $\bar{u}$ is the value of $\delta \bar{\omega}$ evaluated at either of the poles of the event horizon, with $\delta \bar{\omega}$ being the difference between the function $\bar{\omega}$ for the deformed black hole (whether the deformation is due to compactification as in our case, or to external matter) and the function ${ }^{0} \bar{\omega}$ for the undeformed RNN solution with the same $M$, $Q$ and $l$. Since as $\bar{\omega} \rightarrow-\infty$, we have

$$
f=\frac{2 \Delta}{\sqrt{r_{+}^{2}+l^{2}}} e^{\bar{\omega}}+O\left(e^{3 \bar{\omega}}\right)
$$

it follows that $\bar{u}$ is closely related to the change in horizon pole redshift factor that the deformation produces. In the Schwarzschild case $Q=l=0$, the relation is more direct since $f=e^{\bar{\omega}}$ in that case. The term "horizon redshift factor" must not be taken too literally, however, since it normally refers to an asymptotically flat situation when the value of $f^{-1}$ gives the redshift factor between the point of evaluation and infinity where $f=1$. Anyhow, we shall refer to $\bar{u}$ as the redshift parameter. In table we display how $\bar{u}$ has in effect been fixed by previous workers that have studied the compactified Schwarzschild solution. As we see no reason why any one of these choices should be better than the other two, we prefer to keep $\bar{u}$ as a free parameter.

Other quantities that are interesting to calculate on the horizon are the surface gravity $\kappa$ and Komar mass

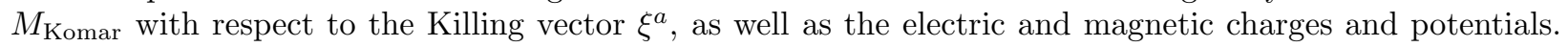
We find that these quantities are given by

$$
\begin{aligned}
& \kappa=\frac{\Delta}{\mathcal{R}^{2}} \\
& M_{\text {Komar }}=\Delta \\
& Q_{\mathrm{el}}=Q \frac{r_{+}^{2}-l^{2}}{r_{+}^{2}+l^{2}} \\
& Q_{\mathrm{mag}}=\frac{2 Q l r_{+}}{r_{+}^{2}+l^{2}} \\
& v=-\frac{Q r_{+}}{r_{+}^{2}+l^{2}} \\
& u=\frac{Q l}{r_{+}^{2}+l^{2}}
\end{aligned}
$$

We note that the surface gravity is constant over the event horizon and thus the zeroth law of thermodynamics holds. Moreover the electric and magnetic potentials are constant as well. In fact the electromagnetic charges and potentials take the exact same values as in the noncompactified case. For a discussion of further thermodynamic properties, we refer the reader to the general framwork for distorted charged black holes [27, 33]. Unfortunately, to our knowledge no such framework exists in the case of nonzero NUT charge, but providing it here would be beyond the scope of this paper. 


\begin{tabular}{|c|c|}
\hline workers & $\bar{u}$ \\
\hline Myers [3] & $\frac{1}{2} \ln \left[2 \varphi_{\beta}(2)\right]+\ln \left[\frac{\Gamma(1-\beta / 2)}{\Gamma(1+\beta / 2)}\right]$ \\
\hline Korotkin and Nicolai [4, 5] & $\frac{1}{2} \ln \left[2 \varphi_{\beta}(2)\right]+\beta \gamma$ \\
\hline Frolov and Frolov [6] & $\frac{1}{2} \ln \left[2 \varphi_{\beta}(2)\right]+\beta \ln (4 \pi)$ \\
\hline
\end{tabular}

TABLE I: The redshift parameter $\bar{u}$ as used in previous studies of the compactified Schwarzschild solution.

\section{Proper distance between black hole poles}

A quantity that characterizes the off-horizon section of the symmetry axis is the proper spatial separation between the poles of the black hole. It can be calculated as

$$
L_{\mathrm{sep}}=2 \Delta \int_{1}^{\beta^{-1}} f^{-1} d y
$$

with $f$ evaluated at $x=0$. To get a general idea of how this quantity depends on the choice of charges $M, Q$ and $l$, we have held the quotients $Q / M$ and $l / M$ fixed at four different values and, with the redshift parameter $\bar{u}$ set to zero, plotted $L_{\mathrm{sep}} / L$ as a function of $\beta$ (figure 2). Since $L_{\text {sep }}$ depends nontrivially on how one chooses $\bar{u}$ to depend on $\beta$, our plot for the case $Q=l=0$ looks different than the one given by Frolov and Frolov [6]. In particular, in [6] $L_{\text {sep }}$ tends to a finite value rather than zero as $\beta \rightarrow 1$, which is counter-intuitive since in that limit the polar points reach each other as the event horizon fills the whole axis. Again, this is directly related to the behaviour of $\bar{u}$ in the same limit. However, any choice of $\bar{u}$ which stays finite results in a vanishing $L_{\mathrm{sep}}$ as $\beta \rightarrow 1$, just like for $\bar{u} \equiv 0$. To show the effect of different choices of $\bar{u}$, we have also plotted $L_{\text {sep }}$ as a function of $\beta$ for the different choices of $\bar{u}$ collected in table \ with the choice $\bar{u} \equiv 0$ again included for comparison (figure 3 ).

\section{Large distance asymptotics}

In the limit $\rho \rightarrow \infty$, our compactified black hole solution approaches an exact solution corresponding to the field of a homogeneous line mass with electric as well as NUT line charge. This solution is obtained simply by truncating all terms that fall off exponentially with $\rho$, which is easy to do given the fourier expansions derived in subsection IVA Explicitly,

$$
\begin{aligned}
\bar{\omega} & =\bar{u}-\frac{1}{2} \ln \left[2 \varphi_{\beta}(2)\right]+\beta \ln \left(\frac{\rho}{2 L}\right) \\
k & =k_{*}+\beta^{2} \ln \left(\frac{\rho}{\Delta}\right) \\
\Omega & =\frac{4 l z}{L}+\Omega^{\prime}
\end{aligned}
$$

so the spacetime metric for our compactified Reisser-Nordström-NUT solution, in the nonextremal case $\Delta>0$, rapidly approaches

$$
d s^{2}=-f^{2}(d t+\Omega d \phi)^{2}+f^{-2}\left[\tilde{C}^{2}\left(\rho / \rho_{0}\right)^{2 \beta^{2}}\left(d \rho^{2}+d z^{2}\right)+\rho^{2} d \phi^{2}\right]
$$

where

$$
f^{2}=\frac{\left(r_{+}-r_{-}\right)^{2}}{\left[r_{-}\left(\rho / \rho_{0}\right)^{\beta}-r_{+}\left(\rho / \rho_{0}\right)^{-\beta}\right]^{2}+l^{2}\left[\left(\rho / \rho_{0}\right)^{\beta}-\left(\rho / \rho_{0}\right)^{-\beta}\right]^{2}}
$$




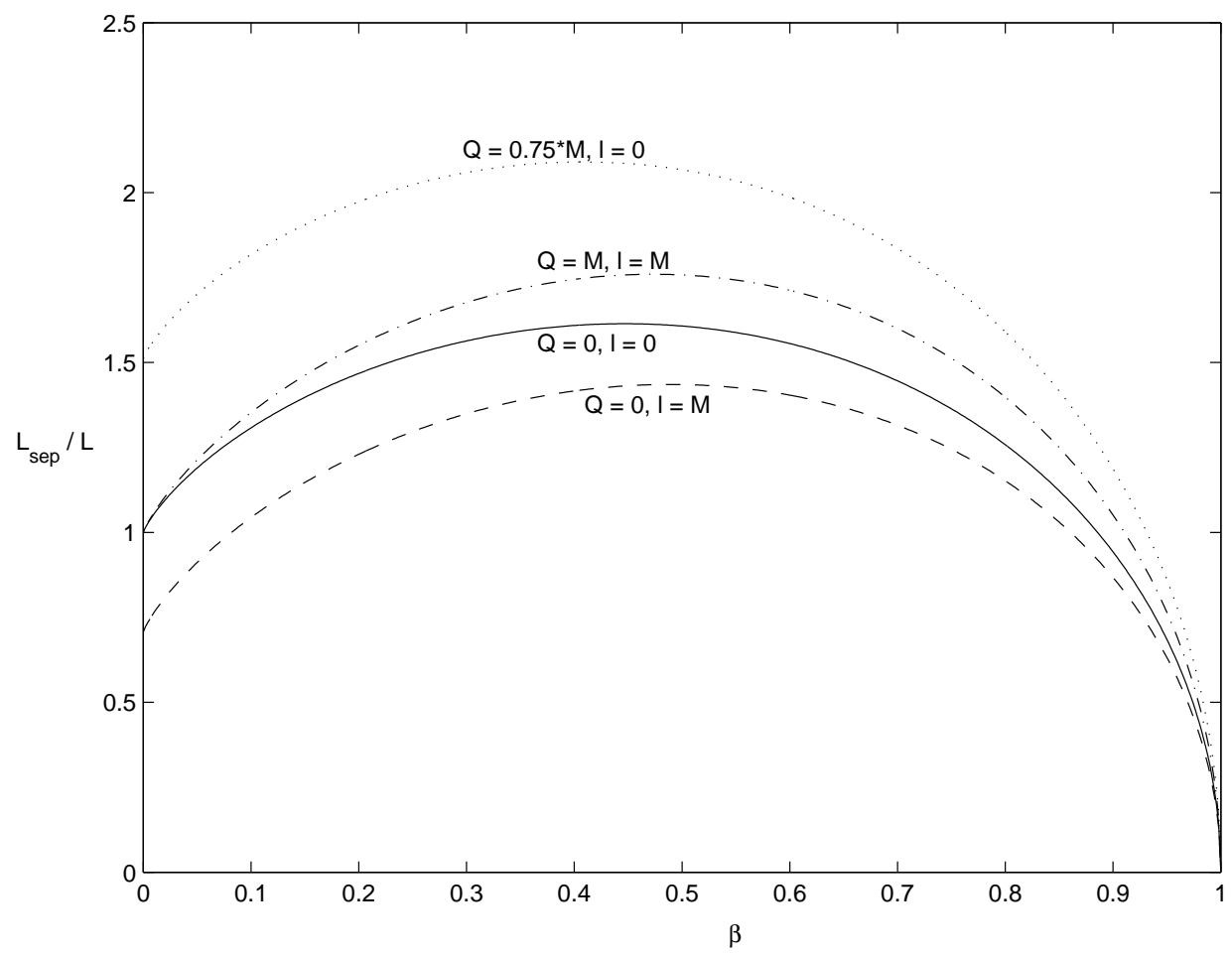

FIG. 2: Proper distance between black hole poles as a function of the parameter $\beta$, for $\bar{u} \equiv 0$ and four particular choices of the quotients $Q / M$ and $l / M$.

As before, $r_{ \pm}=M \pm \Delta$ with $\Delta=\sqrt{M^{2}+l^{2}-Q^{2}}$, but we have here also introduced the constants

$$
\begin{aligned}
& \rho_{0}=\left[2 \varphi_{\beta}(2) e^{-2 \bar{u}}\right]^{\frac{1}{2 \beta}} 2 L \\
& \tilde{C}=\left[\frac{\varphi_{\beta}(2) e^{2 \bar{u}}}{2}\right]^{-\frac{\beta}{2}} e^{\beta^{2}+\int_{0}^{\beta} \ln \left[\varphi_{\beta^{\prime}}(2)\right] d \beta^{\prime}} .
\end{aligned}
$$

If we set both the electric charge $Q$ and NUT charge $l$ to zero, the function $f^{2}$ simplifies to

$$
f^{2}=\left(\frac{\rho}{\rho_{0}}\right)^{2 \beta}
$$

which shows that this is a special case of Levi-Civita's static cylindrically symmetric vacuum solution. As discussed for instance by Bičák et al. [29], the Levi-Civita metric contains two essential parameters $m$ and $\mathcal{C}$ and can be written in the standard form

$$
d s^{2}=-\check{\rho}^{2 m} d \check{t}^{2}+\check{\rho}^{2 m(m-1)}\left(d \check{\rho}^{2}+d \check{z}^{2}\right)+\frac{1}{\mathcal{C}^{2}} \check{\rho}^{2(1-m)} d \phi^{2},
$$

where the coordinates $\check{t}, \check{\rho}$ and $\check{z}$ as well as the conicity parameter $\mathcal{C}$ in general carry dimension in a nonstandard way. The conicity parameter can obviously be set to unity by rescaling the coordinate $\phi$ as $\phi \rightarrow \mathcal{C} \phi$, but since we insist that $\phi$ be a periodic coordinate with the standard range $\phi \in[0,2 \phi)$, the value of $\mathcal{C}$ cannot be viewed as a coordinate choice. In fact, since we are dealing with a compactified spacetime for which $z$ is also a periodic coordinate with a fixed range, the general static cylindrically symmetric solution - what one might call the compactified Levi-Civita solution - has instead three essential parameters, which for instance can be taken to be our $\beta, \rho_{0}$ and $\tilde{C}$, with $\beta$ directly corresponding to $m$. 


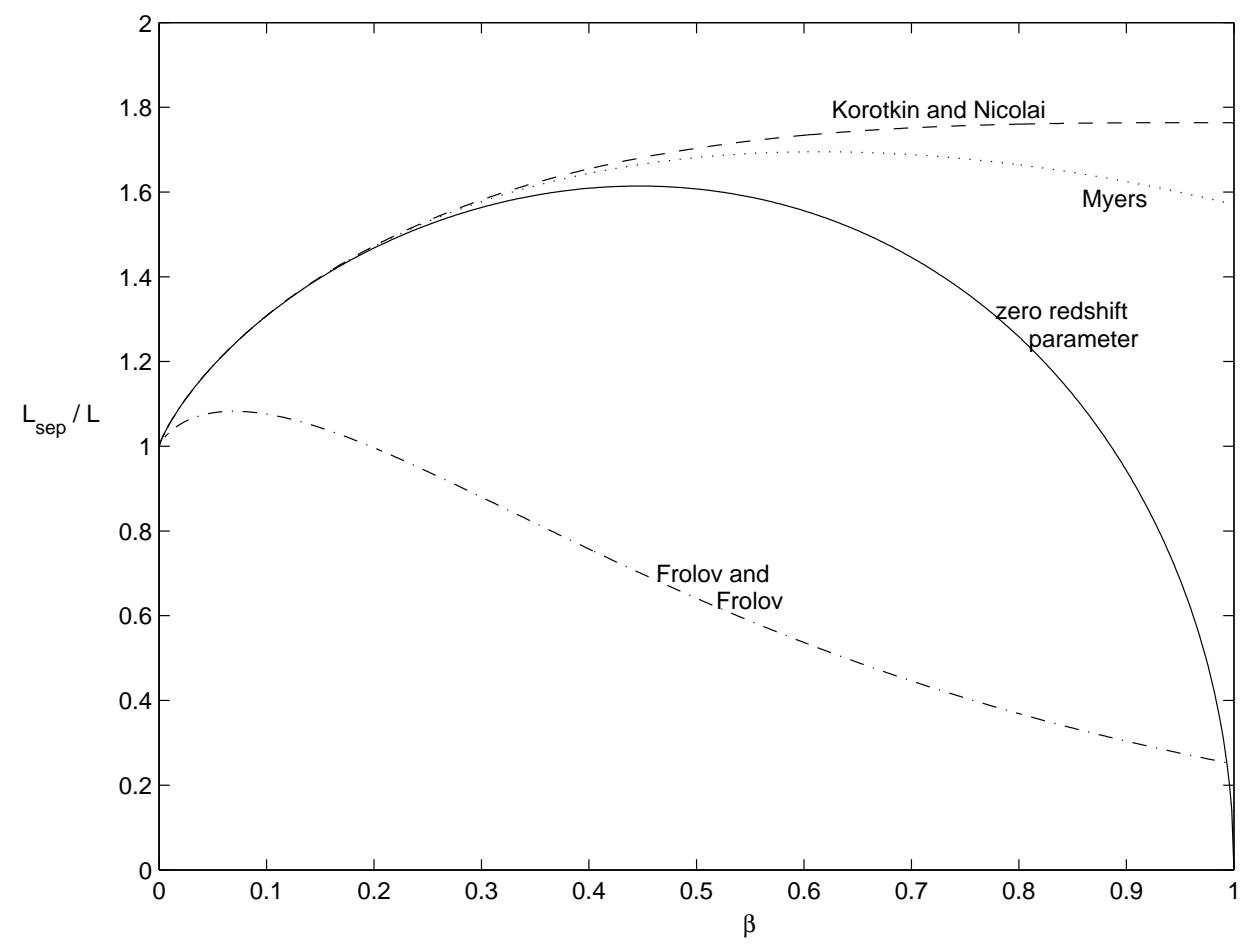

FIG. 3: Proper distance between black hole poles as a function of the parameter $\beta$, for $Q=l=0$ and four different choices of the redshift parameter $\bar{u}$, three of which being taken from the previous studies of the compactified Schwarzschild solution.

Setting the electric charge $Q$ to zero while having a non-vanishing NUT charge $l$ makes it possible to reexpress $f^{2}$ as

$$
f^{2}=\frac{2 \Delta}{r_{+}\left(\rho / \rho_{0}\right)^{2 \beta}-r_{-}\left(\rho / \rho_{0}\right)^{-2 \beta}}=\frac{\Delta}{l} \frac{1}{\cosh \left[2 \beta \ln \left(\rho / \rho_{1}\right)\right]},
$$

where (note that $r_{-}<0$ in this case)

$$
\rho_{1}=\left(-\frac{r_{-}}{r_{+}}\right)^{\frac{1}{4 \beta}} \rho_{0}
$$

As should be expected, this vacuum solution is the "cylindrical analogue of NUT space" that was constructed and studied by Nouri-Zonoz [32]. According to Nouri-Zonoz, the "gravitomagnetic charge per unit length" is (up to sign) equal to the coefficient of $z$ in the expression for $\Omega$, i.e. in our case $4 l / L$. However, what we have constructed here is the asymptotic field of a black hole with NUT charge $l$ in a space with a compactified dimension of ( $z$-coordinate) length $L$, so we expect the NUT charge per unit $z$-length to be $l / L$. We believe that the discrepancy of a factor of 4 is only a matter of definitions (i.e. Nouri-Zonoz' "gravitomagnetic charge" is four times NUT charge, when the latter is definied so that its value for the RNN metric (32) is $l$.

Setting $l=0$ but keeping a non-zero electric charge $Q$, the general asymptotic solution (122) instead becomes the cylindrically symmetric Einstein-Maxwell solution with a purely electric Maxwell field. This is one of the solutions constructed and studied by Richterek et al. [30] as well as Miguelote et al. [31]. The function $f^{2}$ can in this case be written as

$$
f^{2}=\frac{\Delta^{2}}{Q^{2}} \frac{1}{\sinh ^{2}\left[\beta \ln \left(\rho / \rho_{2}\right)\right]}, \quad \rho_{2}=\left(\frac{r_{+}}{r_{-}}\right)^{\frac{1}{2 \beta}} \rho_{0} .
$$


Note that $f^{2}$ diverges at a finite cylinder radius $\rho=\rho_{2}$, indicating that there should be a curvature singularity there. This is indeed the case, as noticed in [30, 31], but let us see this explicitly by calculating the energy density of the electromagnetic field with respect to the unit timelike vector field $u^{a}=f^{-1} \xi^{a}$ where $\xi^{a}=(\partial / \partial t)^{a}$, as before. The result, in the general case with all three charges $M, Q$ and $l$ arbitrary, is

$$
\mu_{\mathrm{EM}}=u^{a} u^{b} T_{a b}=\frac{1}{2} u^{a} u^{b} G_{a b}=2\left(\frac{Q}{\tilde{C} \rho_{0} L}\right)^{2}\left(\rho / \rho_{0}\right)^{-2\left(1+\beta^{2}\right)} f^{4} .
$$

Clearly $\mu_{\mathrm{EM}}$ diverges at finite $\rho$ exactly when $f^{2}$ does. However, the curvature singularity at $\rho=\rho_{2}$ disappears as soon as a NUT charge $l$ is turned on, since the denominator of the right hand side of eq. (123) is then the sum of two squares which cannot vanish simultaneously. Of course, one can make the curvature grow arbitrarily large close to $\rho=\rho_{2}$ by choosing the quotient $l / Q$ sufficiently small. This feature is not only present in the asymptotic solution discussed here, but also in the full compactified RNN solution with the $z$-dependent fourier terms turned back on. The reason for this is that the range of the function $\omega$ changes from $\omega \in(-\infty, 0)$ to $\omega \in(-\infty, \infty)$ when we compactify the spacetime by making the coordinate $z$ periodic; In both cases $\omega \rightarrow-\infty$ occurs at the event horizon, i.e. at $\rho=0,|z| \leq \Delta$, but $\rho \rightarrow \infty$ sends $\omega$ to $+\infty$ rather than zero in the compactified case. In particular, for $Q \neq 0=l$, the compactified $\bar{\omega}$ will take on the positive value

$$
\bar{\omega}_{\text {crit }}=\frac{1}{2} \ln \left(\frac{r_{+}}{r_{-}}\right)
$$

for which $f^{2}$ diverges, as can be seen from eq. (41). Again, this gives a curvature singularity which however is regularized by turning on a NUT charge $l$, however small. The difference, compared to the asymptotic solution, is that the curvature singularity now occurs on some torus $\rho=f(z)$ which is not a simple flat torus $\rho=$ constant. The asymptotic solution also has a curvature singularity on the axis $\rho=0$ for $\beta \in(0,1)$, but this is in contrast to the full compactified RNN solution which by construction is regular on that axis. Another interesting feature related to the NUT parameter $l$ is that although the spacetime metric (122) is cylindrically symmetric in the sense that no physically measurable quantities depend on the $z$, this coordiate still appears in the expression (121) for $\Omega$. What is interesting is that if we demand that the metric be explicitly periodic in $z$ with period $L$, then the metric must be unchanged under $\Omega^{\prime} \rightarrow \Omega^{\prime}+4 n l$ for any integer $n$. This is true, granted that $t$ is periodic with period $8 \pi l$ (just like for the non-compactified RNN solution we started out with), since we can mimick the shift in $\Omega^{\prime}$ by letting $t \rightarrow t+4 n l \phi$.

Turning finally to the extremal case $\Delta=0$, we must use $\omega=M \bar{\omega} / \Delta$ instead of $\bar{\omega}$ before taking the limit $\Delta \rightarrow 0$. The limit then gives

$$
\begin{aligned}
& \omega=\frac{2 M}{L}\left[u^{\prime}+\gamma+\ln \left(\frac{\rho}{2 L}\right)\right], \quad u^{\prime}=\lim _{\beta \rightarrow 0} \beta^{-1} \bar{u} \\
& k=0 \\
& \Omega=\frac{4 l z}{L}+\Omega^{\prime},
\end{aligned}
$$

so the asymptotic spacetime metric is in this case

$$
d s^{2}=-f^{2}(d t+\Omega d \phi)^{2}+f^{-2}\left(d \rho^{2}+d z^{2}+\rho^{2} d \phi^{2}\right),
$$

with, using eq. 477,

$$
f^{2}=\frac{1}{\left[1-\frac{2 M}{L} \ln \left(\rho / \rho_{3}\right)\right]^{2}+\left[\frac{2 l}{L} \ln \left(\rho / \rho_{3}\right)\right]^{2}}, \quad \rho_{3}=2 L e^{-u^{\prime}-\gamma} .
$$

Just like in the nonextremal case, this asymptotic solution fails to be asymptotically flat, albeit in a weaker manner. Also, the discussion concerning the curvature singularity at finite $\rho$ applies here as well. In particular, from eq. (47) it follows that if $l=0$, the asymptotic solution has a curvature singularity at $\rho=\rho_{3} e^{L /(2 M)}$, but the singularity is removed when the NUT charge $l$ is turned back on. 


\section{CONCLUSIONS}

Having constructed and analyzed what we consider to be the natural periodic analogue of the ReissnerNordström-NUT solution, a question that naturally arises is whether or not any given asymptotically flat black hole has a periodic analogue and, if so, whether or not the periodic analogue is unique, given the period $L$ of the compactified coordinate. By identifying the target space geodesic that the non-compactified solution defines and assuming that the very same geodesic should be used for the compactified version, we have found that the problem essentially reduces to finding the appropriate axisymmetric solution to the Laplace equation in flat space, just as in the Schwarzschild case. There is thus a simple superposition principle at play, which makes compactification tractable when Weyl coordinates are used. However, the low dimension requires a regularization procedure which introduces a one-parameter ambiguity as there is no obvious way to fix the freedom to add an arbitrary constant to the solution to the Laplace equation. In all previous works on the periodic Schwarzschild solution, this constant - the redshift parameter - was always fixed at an early stage and without any physical motivation for the specific choice made. As we have seen there are three different choices in the existing papers on the subject, which we take as support for instead keeping the parameter free, or at least fixing it at a later stage from physical considerations. One way of fixing it would be to require that a set of relations that hold for horizon quantities (for instance the relation between the horizon area and the black hole charges) should remain the same after compactification. If one could motivate why this should be the case, our method would give a neat one-to-one correspondance between standard and periodic Reissner-Nordström-NUT black holes. Of course, we have not given any proof that our method is correct, in some suitably defined sense. One could perhaps argue that it is not, based on the fact that our solution family has a curvature singularity surrounding the horizon in the case of electric but no NUT charge. However, we see no way of avoiding such a singularity. Indeed, the singularity in question is actually a general feature not just for our full solution with a nontrivial dependence on the periodic coordinate, but also for the cylindrically symmetric solution that is approached for large cylinder radii. Since there simply is no other cylindrically symmetric solution available that could work as an asymptotic solution for a compactified Reissner-Nordström black hole, it seems at least likely that the exponentially decaying corrections to the asymptotic solution cannot smooth out the singularity.

One can think of several ways to extend our work while staying in four dimensions. One way would be to include a dilaton field with arbitrary coupling parameter. This would be straightforward to do, as our method would still be applicable with the dilaton simply entering as an extra target space coordinate. It would be interesting to see whether the inclusion of a dilaton could prevent the above-mentioned singularity from occuring. A general framework for distorted charged dilaton black holes has been provided by Yazadjiev 33 .

It would be even more interesting if the periodic analogue of the Kerr solution could be constructed. Some indications of how this could be done were given in [5], but no explicit formulae were given there, except for a few first steps. Since the noncompactifed Kerr solution traces out a target space two-surface rather than a geodesic, it would no longer be possible to linearize the problem and thus more sophisticated methods, such as Bäcklund transformations, are needed.

It would of course be even more interesting if our work could give some insights into how things work in higher dimensions. Consider the Einstein-Maxwell equations for a static $(d+1)$-dimensional spacetime. Dimensionally reducing with respect to the static Killing vector, one arrives at Einstein gravity in $d$ euclidean dimensions, coupled to a two-dimensional (three-dimensional if a dilaton is included) sigma model. One question one could ask is whether or not the target space geodesic that the $(d+1)$-dimensional ReissnerNordström solution corresponds to would also be traced out when the solution is compactified on a circle. The work of Harmark and Obers actually suggests that this could be the case, as their ansatz is such that the target space coordinates are functionally dependent, thus corresponding to geodesic solutions. This does not mean that it would be easy to make analytical progress for $d>3$, but nevertheless it could give a coordinate invariant way of thinking about the ansatz of these authors which in turn could lead to a deeper understanding of the general problem. It would be very interesting to investigate this matter further. 


\section{Acknowledgements}

The work of RvU was supported by the Ministry of Education of the Czech Republic under the project MSM 0021622409. The work of MK was supported by a post doctoral fellowship of the Faculty of Science of Masaryk University.

[1] S. D. Majumdar, “A Class Of Exact Solutions Of Einstein's Field Equations," Phys. Rev. 72 (1947) 390.

[2] A. Papapetrou, Proc. R. Irish Acad. A51, (1947) 191.

[3] R. C. Myers, "Higher Dimensional Black Holes In Compactified Space-Times," Phys. Rev. D 35 (1987) 455.

[4] D. Korotkin and H. Nicolai, "A Periodic analog of the Schwarzschild solution," arXiv:gr-qc/9403029

[5] D. Korotkin and H. Nicolai, "The Ernst equation on a Riemann surface," Nucl. Phys. B 429 (1994) 229 arXiv:gr-qc/9405032.

[6] A. V. Frolov and V. P. Frolov, "Black holes in a compactified spacetime," Phys. Rev. D 67 (2003) 124025 arXiv:hep-th/0302085.

[7] R. Gregory and R. Laflamme, "Black strings and p-branes are unstable," Phys. Rev. Lett. 70 (1993) 2837 arXiv:hep-th/9301052.

[8] R. Gregory and R. Laflamme, "The Instability of charged black strings and p-branes," Nucl. Phys. B 428 (1994) 399 arXiv:hep-th/9404071.

[9] G. T. Horowitz and K. Maeda, "Fate of the black string instability," Phys. Rev. Lett. 87 (2001) 131301 arXiv:hep-th/0105111.

[10] T. Harmark and N. A. Obers, "Black holes on cylinders," JHEP 0205 (2002) 032 arXiv:hep-th/0204047.

[11] T. Harmark and N. A. Obers, "New phase diagram for black holes and strings on cylinders," Class. Quant. Grav. 21 (2004) 1709 arXiv:hep-th/0309116.

[12] T. Harmark and N. A. Obers, "Phase structure of black holes and strings on cylinders," Nucl. Phys. B 684 (2004) 183 arXiv:hep-th/0309230.

[13] T. Harmark "Small black holes on cylinders," Phys. Rev. D 69 (2004) 104015 arXiv:hep-th/0310259.

[14] H. Elvang, T. Harmark and N. A. Obers, "Sequences of bubbles and holes: New phases of Kaluza-Klein black holes," JHEP 0501 (2005) 003 arXiv:hep-th/0407050.

[15] S. S. Gubser, "On non-uniform black branes," Class. Quant. Grav. 19 (2002) 4825 arXiv:hep-th/0110193.

[16] B. Kol, "Topology change in general relativity and the black-hole black-string transition," arXiv:hep-th/0206220

[17] B. Kol and T. Wiseman, "Evidence that highly non-uniform black strings have a conical waist," Class. Quant. Grav. 20 (2003) 3493 arXiv:hep-th/0304070.

[18] B. Kol, E. Sorkin and T. Piran, "Caged black holes: Black holes in compactified spacetimes. I: Theory," Phys. Rev. D 69 (2004) 064031 arXiv:hep-th/0309190.

[19] E. Sorkin, B. Kol and T. Piran, "Caged black holes: Black holes in compactified spacetimes. II: 5d numerical implementation," Phys. Rev. D 69 (2004) 064032 arXiv:hep-th/0310096.

[20] D. Gorbonos and B. Kol, "A dialogue of multipoles: Matched asymptotic expansion for caged black holes," JHEP 0406 (2004) 053 arXiv:hep-th/0406002.

[21] D. Gorbonos and B. Kol, "Matched asymptotic expansion for caged black holes: Regularization of the postNewtonian order," arXiv:hep-th/0505009

[22] T. Wiseman, "Static axisymmetric vacuum solutions and non-uniform black strings," Class. Quant. Grav. 20 (2003) 1137 arXiv:hep-th/0209051.

[23] T. Wiseman, "From black strings to black holes," Class. Quant. Grav. 20 (2003) 1177 arXiv:hep-th/0211028.

[24] H. Kudoh and T. Wiseman, "Properties of Kaluza-Klein black holes," Prog. Theor. Phys. 111 (2004) 475 arXiv:hep-th/0310104.

[25] H. Kudoh and T. Wiseman, "Connecting black holes and black strings," Phys. Rev. Lett. 94 (2005) 161102 arXiv:hep-th/0409111.

[26] R. Emparan and H. S. Reall, "Generalized Weyl solutions," Phys. Rev. D 65 (2002) 084025 arXiv:hep-th/0110258.

[27] S. Fairhurst and B. Krishnan, "Distorted black holes with charge," Int. J. Mod. Phys. D 10 (2001) 691 arXiv:gr-qc/0010088.

[28] R. Geroch and J. B. Hartle, "Distorted black holes," Jour. Math. Phys. 23 (1981) 680

[29] J. Bicak, T. Ledvinka, B. G. Schmidt and M. Zofka, "Static fluid cylinders and their fields: global solutions," Class. Quant. Grav. 21 (2004) 1583 arXiv:gr-qc/0403012.

[30] L. Richterek, J. Novotný and J. Horský, "New Einstein-Maxwell fields of Levi-Civita's type," Czech. J. Phys. 
$50(2000) 925$ arXiv:gr-qc/0003004.

[31] A. Y. Miguelote, M. F. A. da Silva, A. Wang and N. O. Santos, "Levi-Civita solutions coupled with electromagnetic fields," Class. Quant. Grav. 18 (2001) 4569 arXiv:gr-qc/0104018.

[32] M. Nouri-Zonoz, "Cylindrical analogue of NUT space: Spacetime of a line gravomagnetic monopole," Class. Quant. Grav. 14 (1997) 3123 arXiv:gr-qc/9706015.

[33] S. S. Yazadjiev, "Distorted charged dilaton black holes," Class. Quant. Grav. 18 (2001) 2105 arXiv:gr-qc/0012009.

[34] We use units such that the four-dimensional Einstein-Maxwell equations read $G_{a b}=2 T_{a b}$ where $T_{a b}=F_{a}{ }^{c} F_{b c}-$ $\frac{1}{4} F^{c d} F_{c d} g_{a b}$. 